\title{
Direct Oral Anticoagulants for Pulmonary Embolism: Importance of Anatomical Extent
}

\author{
Marjolein P. A. Brekelmans ${ }^{1}$ Harry R. Büller ${ }^{1}$ Michele F. Mercuri ${ }^{2}$ Walter Ageno ${ }^{3}$ Cathy Z. Chen ${ }^{4}$ \\ Alexander T. Cohen ${ }^{5}$ Nick van Es ${ }^{1}$ Michael A. Grosso ${ }^{2}$ Andria P. Medina ${ }^{6}$ Gary Raskob ${ }^{7}$ \\ Annelise Segers ${ }^{8}$ Thomas Vanassche ${ }^{9}$ Peter Verhamme ${ }^{9} \quad$ Philip S. Wells $^{10}$ George Zhang ${ }^{2}$ \\ Jeffrey I. Weitz ${ }^{11}$ \\ 1 Department of Vascular Medicine, Academic Medical Center, \\ Amsterdam, The Netherlands \\ ${ }^{2}$ Clinical Development, Daiichi Sankyo Pharma Development, \\ Edison, New Jersey, United States \\ ${ }^{3}$ Department of Clinical and Experimental Medicine, University of \\ Insubria, Varese, Italy \\ ${ }^{4}$ Global Medical Affairs, Daiichi Sankyo Inc., Parsippany, \\ New Jersey, United States \\ ${ }^{5}$ Department of Haematological Medicine, Guy's and St Thomas' \\ Hospitals, King's College London, London, United Kingdom \\ ${ }^{7}$ College of Public Health, University of Oklahoma Health Sciences \\ Center, Oklahoma City, Oklahoma, United States \\ 8 Itreas BV, Amsterdam, The Netherlands \\ ${ }^{9}$ Vascular Medicine and Haemostasis, University of Leuven, \\ Leuven, Belgium \\ ${ }^{10}$ Department of Medicine, University of Ottawa, Ottawa Hospital \\ Research Institute, Ottawa, Canada \\ ${ }^{11}$ Thrombosis and Atherosclerosis Research Institute and McMaster \\ University, Hamilton, Ontario, Canada
}

${ }^{6}$ Department of Medicine, University of Oklahoma Health Sciences Center, Oklahoma City, Oklahoma, United States

TH Open 2018;2:e1-e7.

Address for correspondence Marjolein P. A. Brekelmans, MD, Department of Vascular Medicine, Academic Medical Center, Meibergdreef 9, 1105 AZ, Amsterdam, The Netherlands (e-mail: m.p.brekelmans@amc.uva.nl).

\begin{abstract}
Keywords

- pulmonary embolism

- venous thromboembolism

- right ventricular dysfunction

- oral anticoagulants

- anatomical extent

Pulmonary embolism (PE) studies used direct oral anticoagulants (DOACs) with or without initial heparin. We aimed to (1) evaluate if PE patients benefit from initial heparin; (2) describe patient characteristics in the DOAC studies; and (3) investigate whether the anatomical extent of $\mathrm{PE}$ correlates with $\mathrm{N}$-terminal pro-brain natriuretic peptide (NT-proBNP) levels, cause of PE, and recurrence rate. Our methods were (1) an indirect meta-analysis comparing the recurrence risk in DOAC-treated patients with or without initial heparin to those patients given heparin/vitamin K antagonist (VKA). (2) To compare the PE studies, information was extracted on baseline characteristics including anatomical extent. (3) The Hokusai-VTE study was used to correlate anatomical extent of PE with NT-proBNP levels, causes of PE, and recurrent venous thromboembolism (VTE). The meta-analysis included 11,539 PE patients. The relative risk of recurrent VTE with DOACs versus heparin/VKAs was 0.8 (95\% confidence interval [Cl]: 0.6-1.1) with heparin lead-in and 1.1 (95\% Cl: 0.8-1.5) without heparin. In the DOAC studies, the proportion of patients with extensive PE varied from 24 to $47 \%$. In Hokusai-VTE, NT-proBNP was elevated in $4 \%$ of patients with limited and in over $60 \%$ of patients with extensive disease. Cause of PE and anatomical extent were not related. Recurrence rates increased from $1.6 \%$ with limited to $3.2 \%$ with extensive disease in heparin/edoxaban-treated patients, and from 2.4 to $3.9 \%$ in heparin/warfarin recipients. In conclusion, indirect evidence suggests a heparin lead-in before DOACs may be advantageous in PE. Anatomical extent was related to elevated NT-proBNP and outcome, but not to PE cause.
\end{abstract}

received

June 29, 2017

accepted after revision

November 13, 2017
DOI https://doi.org/

10.1055/s-0037-1615251. ISSN 2512-9465.
(C) 2018 Georg Thieme Verlag KG Stuttgart · New York
License terms

(1)(1) 


\section{Introduction}

Direct oral anticoagulants (DOACs) are at least as effective as vitamin K antagonists (VKAs) for treatment of venous thromboembolism (VTE), but are more convenient to administer and are associated with less bleeding. ${ }^{1}$ Because of these attributes, current guidelines give preference to the DOACs over heparin/VKAs for VTE treatment in patients without active cancer. $^{2}$

Studies comparing DOACs with heparin/VKAs have either started the DOAC after a mandatory heparin lead-in of at least 5 days, or have adopted an all-oral DOAC regimen. ${ }^{3-8}$ These approaches have not been directly compared. In all studies, the comparator was standard of care at that time, consisting of a minimum 5-day course of heparin overlapping with a VKA targeted at an international normalized ratio (INR) of 2.0 to 3.0 .

Although all-oral regimens of DOACs are available, clinicians are often reluctant to forgo heparin treatment in patients with pulmonary embolism (PE), particularly in those with computed tomographic (CT) evidence of extensive disease and/or right ventricular enlargement. $^{9-11}$

The first objective of the current analysis was to evaluate whether PE patients may benefit from initial heparin therapy by indirectly comparing the rate of recurrent VTE in studies with and without a mandatory heparin lead-in. The second objective was to describe the characteristics of PE patients across the studies to assess whether relevant differences exist. The third objective was to better understand whether the anatomical extent of PE correlates with the presence of right ventricular dysfunction (RVD), as evidenced by an elevated serum $\mathrm{N}$-terminal pro-brain natriuretic peptide (NT-proBNP) level, the cause of PE, and the rate of recurrence. For the third purpose, we used data from Hokusai-VTE, because this was the only study that prospectively assessed all these variables. ${ }^{8,12}$

\section{Methods}

\section{Objective 1: Recurrent VTE with or without Mandatory Heparin Lead-in}

A meta-analysis of phase 3 randomized controlled trials comparing DOACs with heparin/VKAs for treatment of acute symptomatic VTE was performed. The selection and identification of these studies were part of a previously published study. ${ }^{13}$ We extracted data on the PE patients from RECOVER, RE-COVER II, EINSTEIN-PE, AMPLIFY, and HokusaiVTE. In this analysis, we focused on recurrent VTE, which was defined in a similar fashion in these studies, and compared the rates of recurrence in patients treated with heparin overlapping with a VKA and DOACs with or without a mandatory heparin lead-in.

We calculated relative risks (RR) and corresponding 95\% confidence intervals $(\mathrm{CI})$ for recurrent VTE; results were combined using the Mantel-Haenszel method and compared using the DerSimonian and Laird random-effects model. We assessed and quantified statistical heterogeneity across the studies using the Cochran's $\mathrm{Q}$ and $\mathrm{I}^{2}$ statistics. The analysis was performed using Review Manager, version 5.2 (Copenhagen, The Nordic Cochrane Centre, The Cochrane Collaboration, 2012).

\section{Objective 2: Patient Characteristics in the DOAC Trials} We extracted information on the baseline characteristics of PE patients including age, gender, creatinine clearance, causes of PE (unprovoked, cancer, or temporary risk factors), previous VTE, and anatomical extent of PE from the published DOAC trials. ${ }^{6-8,14}$ In the AMPLIFY study, separate data for the PE cohort regarding age, gender, creatinine clearance, causes of PE, and previous VTE were not provided. ${ }^{7}$ Information on the anatomical extent of PE was unavailable for the RE-COVER and RE-COVER II studies. ${ }^{14}$ For the other three studies, limited extent was defined as involvement of $25 \%$ or less of the vasculature of a single lobe, whereas intermediate extent was defined as involvement of more than $25 \%$ of the vasculature of a single lobe or multiple lobes with involvement of $25 \%$ or less of the entire vasculature. The definition of extensive disease varied; it was defined as involvement of multiple lobes with compromise of $25 \%$ or more of the entire vasculature in EINSTEIN-PE and Hokusai-VTE, 6,8 whereas in AMPLIFY, extensive disease was defined as involvement of two or more lobes compromising $50 \%$ or more of the vasculature of each lobe. ${ }^{7}$ The latter definition may classify more patients as having extensive disease than the former, because it focuses only on the vasculature of the involved lobes rather than the entire vasculature of the lungs.

\section{Objective 3: Anatomical Extent, Right Ventricular Dysfunction, Causes of PE, and Recurrent VTE in the Hokusai-VTE Study}

The Hokusai-VTE study was a randomized, double-blind trial that compared heparin/edoxaban with heparin/warfarin in 8,292 acute, symptomatic VTE patients (ClinicalTrials.gov identifier: NCT00986154). ${ }^{8,12}$ This analysis focuses on the 3,319 patients with PE. Initial treatment consisted of open-label enoxaparin or unfractionated heparin for at least 5 days followed by either edoxaban or warfarin for a minimum of 3 months and a maximum of 12 months. The dose of edoxaban was $60 \mathrm{mg}$ once daily; this was reduced to $30 \mathrm{mg}$ in patients with a creatinine clearance of 30 to $50 \mathrm{~mL} /$ minute, a body weight $60 \mathrm{~kg}$ or less, or in those receiving potent concomitant P-glycoprotein inhibitors such as verapamil or quinidine. Warfarin was started concomitantly with heparin and the heparin was stopped when the INR was 2.0 or higher. The primary efficacy outcome was the incidence of symptomatic recurrent VTE defined as a composite of deep vein thrombosis (DVT) or nonfatal or fatal PE over the 12 months of the study. This outcome was assessed by a central adjudication committee whose members were unaware of the treatment allocation. ${ }^{8}$

In patients with PE, RVD was defined as a NT-proBNP level of $500 \mathrm{ng} / \mathrm{mL}$ or higher. Serum NT-proBNP levels were measured in samples collected at enrolment. Assays were performed centrally in Quintiles Laboratories (Marietta, Georgia, United States) using the Elecsys NT-proBNP electrochemiluminescence kit on the Roche Cobas e411 platform. Data on PE extent were not available for patients with 
NT-proBNP levels of less than $500 \mathrm{ng} / \mathrm{mL}$ or patients who did not have NT-proBNP levels measured. Causes of PE were classified as either unprovoked or associated with cancer or temporary risk factors (i.e., recent surgery, trauma, immobilization, or use of estrogen $)^{8}$ The definitions of cancer used in the studies were accepted.

In the current analysis, we correlated the anatomical extent of PE at baseline with NT-proBNP levels, causes of $\mathrm{PE}$, and recurrent VTE at 12 months of follow-up. The results are presented as number of events and percentages.

\section{Results}

\section{Rates of Recurrent VTE with or without Heparin Lead- in in PE Patients}

-Fig. 1 presents the pooled analyses of results that included 11,539 PE patients. Rates of recurrent VTE in patients treated with DOACs were compared with those in patients given standard-of-care treatment. The RR of recurrent VTE with DOAC treatment versus heparin/VKA was 0.76 (95\% CI: 0.561.05; heterogeneity $I^{2}=0 \%$; recurrence rates 2.7 vs. $3.5 \%$ ) with heparin lead-in and 1.05 (95\% CI: 0.76-1.46; heterogeneity $I^{2}=0 \%$; recurrence rates 2.1 vs. $2.0 \%$ ) without heparin lead-in.

\section{Patient Characteristics across the DOAC Trials}

- Table 1 details the similarities and differences in patient characteristics across the studies based on the published data. Three studies (RE-COVER, RE-COVER II, and HokusaiVTE) had a mandatory heparin lead-in in the DOAC arm. Age and gender were comparable across the studies. The anatomical extent of PE was not determined in the RE-COVER studies. Although the Hokusai-VTE and EINSTEIN-PE studies used an identical definition of anatomical extent, almost $50 \%$ of the patients had extensive PE in the Hokusai-VTE study, whereas a quarter of the patients had extensive PE in the EINSTEIN-PE study. In the AMPLIFY study, approximately 35\% had extensive PE using a different definition. Another difference was the proportion of patients with temporary risk factors, ranging from approximately $16 \%$ in RE-COVER to around $40 \%$ in EINSTEIN-PE.

\section{Associations of Anatomical Extent with Right Ventricular Dysfunction, Causes of PE, and Recurrent VTE in the Hokusai-VTE Study}

Of the 3,319 PE patients in the Hokusai-VTE study, 90\% had NTproBNP levels measured as baseline $(1,484 / 1,650$ in the heparin/edoxaban and 1,505/1,669 in the heparin/warfarin group). In patients receiving heparin/edoxaban, 465 of 1,650 (28\%) had NT-proBNP levels of $500 \mathrm{ng} / \mathrm{mL}$ or higher compared with 507 of 1,669 (30\%) in the heparin/warfarin arm; 1,019 of 1,650 (62\%) of heparin/edoxaban and 998 of 1669 (60\%) of heparin/warfarin recipients had NT-proBNP levels of less than $500 \mathrm{ng} / \mathrm{mL}$. There was an association between anatomical extent of PE and elevated levels of NT-proBNP in both treatment arms; the proportion of patients with NT-proBNP levels of greater than $500 \mathrm{ng} / \mathrm{mL}$ was higher in patients with an intermediate and highest in patients with extensive PE compared with those with limited disease (-Table $\mathbf{2}$ ).

The proportion of patients with unprovoked PE was comparable at approximately $60 \%$ in all three categories of anatomical extent, with similar proportions in the two treatment arms ( - Table 3 ).

In the edoxaban group, there was an association between anatomical extent of $\mathrm{PE}$ and recurrence rate, increasing from $1.6 \%$ with limited extent to $3.2 \%$ with extensive disease. This correlation was less clear in the heparin/warfarin group, because the recurrence rates in patients with intermediate and extensive PE were similar (-Table 4). In fact, the risk reduction with edoxaban appears consistent across the categories of anatomical extent.

\section{Discussion}

Management of PE patients remains challenging, but this study provides new insights that could aid in guiding treatment decisions. There are three main findings. First, the indirect meta-analysis comparing DOAC and heparin/VKA therapy suggests a possible benefit for a heparin lead-in before starting the DOAC, because even though the CIs overlap, the point estimate with a heparin lead-in is lower. Another observation is that the incidence of VTE recurrence was 3.5\% in the control arm of the studies where a heparin lead-in was used with the DOACs, whereas it was $2.0 \%$ in the control arm in the other comparison, suggesting that PE patients at higher risk of recurrence were enrolled in the heparin lead-in studies. The dilemma of whether or not to use heparin before starting a DOAC can only be resolved with a head-to-head comparison.

Second, the cross-study comparison of the PE patients enrolled in the DOAC studies reveals similarities among many of the baseline characteristics such as age, gender, and the proportion of patients with previous VTE. The most notable difference is the proportion of patients with extensive $\mathrm{PE}$ at the time of diagnosis, with $45 \%$ of patients in

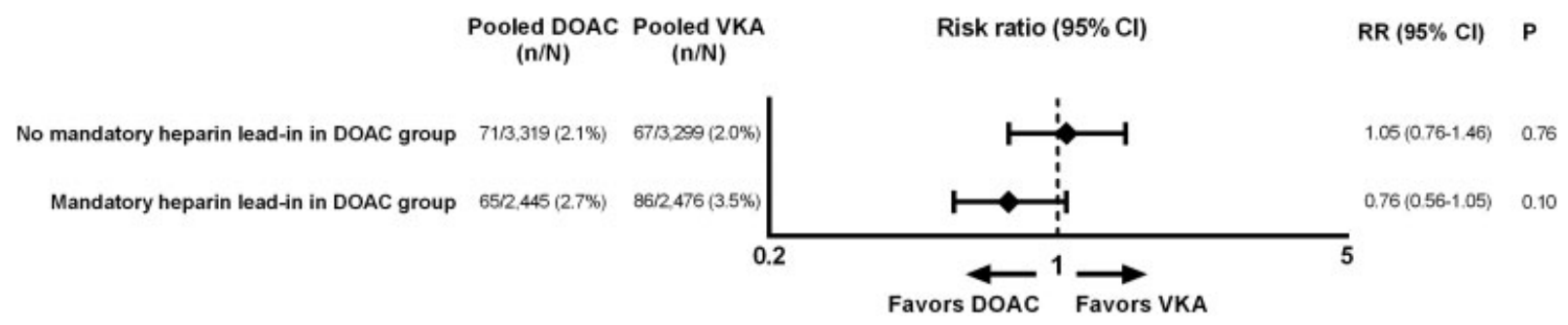

Fig. 1 Relative risk of recurrent venous thromboembolism with or without a heparin lead-in. DOAC, direct oral anticoagulant; VKA, vitamin K antagonist. 


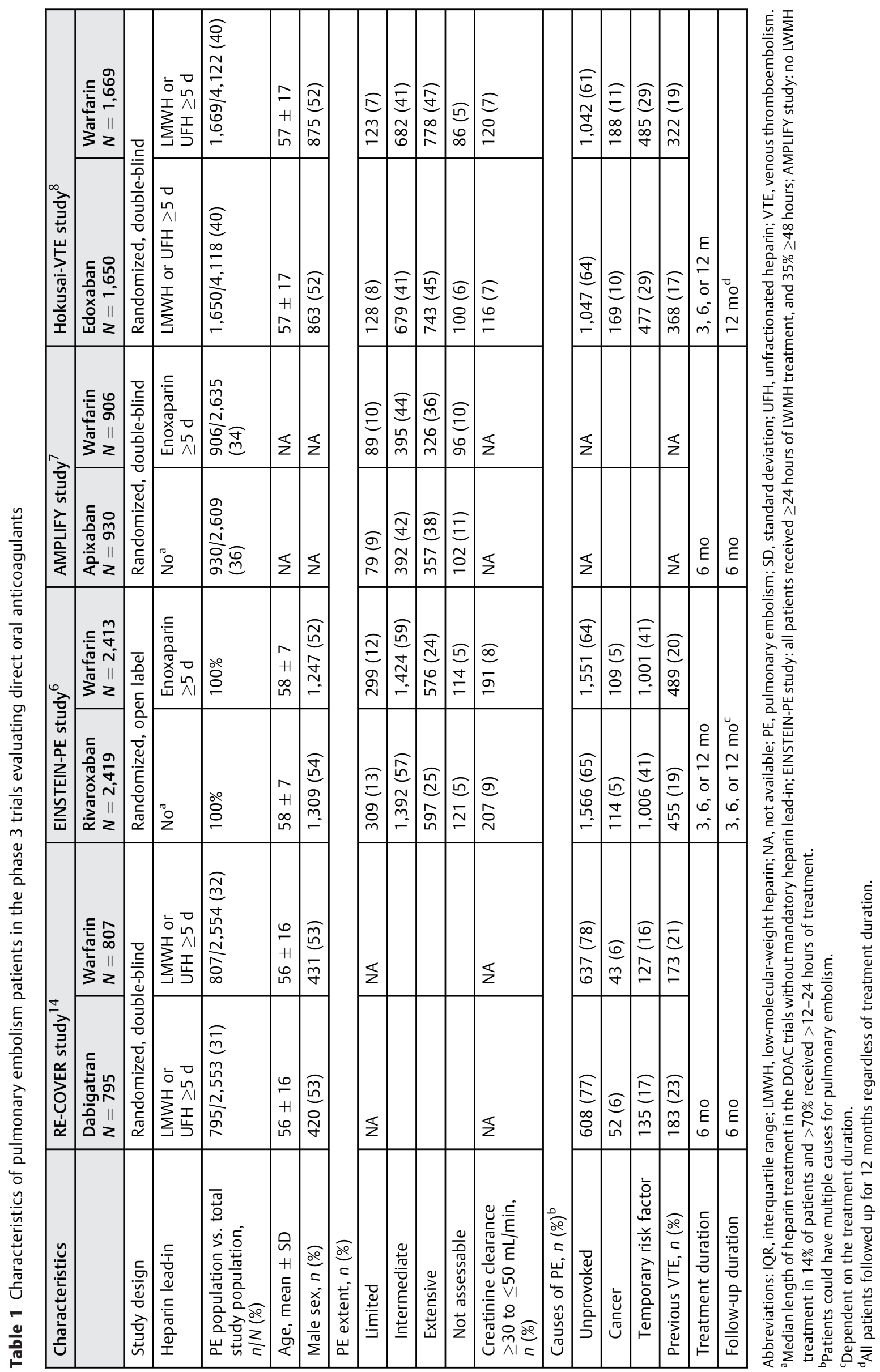


Table 2 Correlation between anatomical extent of PE and elevated NT-proBNP levels

\begin{tabular}{|l|l|l|}
\hline Characteristics & \multicolumn{2}{|l|}{ Hokusai-VTE study } \\
\cline { 2 - 3 } & $\begin{array}{l}\text { Heparin/ } \\
\text { Edoxaban } \\
N=465\end{array}$ & $\begin{array}{l}\text { Heparin/ } \\
\text { Warfarin } \\
N=507\end{array}$ \\
\hline PE extent for patients with NT-proBNP $\geq 500 \mathrm{ng} / \mathrm{mL}, n(\%)$ \\
\hline Limited & $18(4)$ & $22(4)$ \\
\hline Intermediate & $133(29)$ & $136(27)$ \\
\hline Extensive & $293(63)$ & $327(65)$ \\
\hline Not assessable & $21(4)$ & $22(4)$ \\
\hline
\end{tabular}

Abbreviations: NT-pro BNP, N-terminal pro-brain natriuretic peptide; PE, pulmonary embolism; VTE, venous thromboembolism.

Table 3 Anatomical extent of PE and the proportion of patients with unprovoked PE

\begin{tabular}{|l|l|l|}
\hline \multirow{2}{*}{$\begin{array}{l}\text { PE extent and } \\
\text { unprovoked PE }\end{array}$} & \multicolumn{2}{|l|}{ Hokusai-VTE study } \\
\cline { 2 - 3 } & $\begin{array}{l}\text { Heparin/ } \\
\text { Edoxaban } \\
N=1,650\end{array}$ & $\begin{array}{l}\text { Heparin/ } \\
\text { Warfarin } \\
N=1,669\end{array}$ \\
\hline Unprovoked PE per category of PE extent, $n / N(\%)$ \\
\hline Limited & $75 / 128(59)$ & $72 / 123(59)$ \\
\hline Intermediate & $415 / 679(61)$ & $422 / 682(62)$ \\
\hline Extensive & $489 / 743(66)$ & $494 / 778(64)$ \\
\hline Not assessable & $68 / 100(68)$ & $54 / 86(63)$ \\
\hline
\end{tabular}

Abbreviation: PE, pulmonary embolism; VTE, venous thromboembolism.

Hokusai-VTE having extensive PE compared with $25 \%$ in EINSTEIN-PE. A potential explanation for this difference might be the heparin lead-in, because physicians may have been more willing to enroll patients with extensive PE in a study that used a heparin lead-in in all patients.

Third, the in-depth analysis of the Hokusai-VTE study reveals an association between the anatomical extent of PE

Table 4 Correlation between anatomical extent of PE and recurrence

\begin{tabular}{|c|l|l|}
\hline \multirow{2}{*}{ Recurrence rates } & \multicolumn{3}{|l|}{ Hokusai-VTE study } \\
\cline { 2 - 3 } & $\begin{array}{l}\text { Heparin/Edoxaban } \\
N=1,650\end{array}$ & $\begin{array}{l}\text { Heparin/Warfarin } \\
N=1,669\end{array}$ \\
\hline VTE recurrence rate per PE extent, $n / N(\%)$ \\
\hline Limited & $2 / 128(1.6)$ & $3 / 123(2.4)$ \\
\hline Intermediate & $17 / 679(2.5)$ & $27 / 682(4.0)$ \\
\hline Extensive & $24 / 743(3.2)$ & $30 / 778(3.9)$ \\
\hline Not assessed & $4 / 100(4.0)$ & $5 / 86(5.8)$ \\
\hline
\end{tabular}

Abbreviations: PE, pulmonary embolism; VTE, venous thromboembolism. and elevated NT-proBNP levels, a known marker of RVD. ${ }^{15,16}$ Only $4 \%$ of patients with limited disease had an elevated NTproBNP, whereas the proportion with an elevated NTproBNP was $29 \%$ with intermediate and $65 \%$ with extensive disease, findings indicative of a biological gradient. The risk of recurrence was almost twofold higher in patients with extensive disease than in those with limited disease in both treatment arms. Hence, the more patients with extensive disease, the greater the likelihood that the population studied has RVD, a known indicator of an increased risk of recurrent VTE and mortality. ${ }^{17-20}$ Previous studies correlated PE extent to right ventricular enlargement, ${ }^{21}$ and to thrombus load on CT with degree of right ventricular enlargement. ${ }^{22}$ However, to our knowledge, our study is the first to investigate a correlation between anatomical extent of PE and NT-proBNP levels, and recurrent VTE events. Another novel finding is that the proportion of patients with unprovoked PE was comparable in all groups of anatomical extent, suggesting that the extent of the PE is independent of whether the PE is provoked or unprovoked.

A few aspects of the present analyses require comment. The results come from three different approaches: an indirect meta-analysis, a cross-study comparison, and a further in-depth analysis of data from the Hokusai-VTE study. However, all of the data come from well-executed, randomized, controlled trials. The results of the meta-analysis need to be interpreted with caution, because they are based on an indirect comparison. In addition, the observed difference in the point estimate in recurrent VTE between the two approaches (with or without heparin) may also be due to unknown differences between the study populations. Furthermore, an informal analysis of heparin lead-in among patients with DVT only appeared not to support a potential benefit of initial heparin (data not shown). Nonetheless, the findings are intriguing and require further investigation. Another remark is that since the Hokusai-VTE study was the last of the phase 3 DOAC trials, it had the advantage of taking some methodological issues from the previous studies a priori into account. The Hokusai-VTE study was the first to prospectively collect patient data on right ventricular dysfunction, as assessed by NT-proBNP levels and right ventricular/left ventricular ( $\mathrm{RV} / \mathrm{LV})$ diameter ratio on $\mathrm{CT}$, whereas the other studies did not. NT-proBNP levels were measured at the time of enrolment in the study, whereas anatomical extent of PE was measured at the time of diagnosis, and although the time between the diagnosis and inclusion in the study was only a couple of hours in the majority of patients, this may have influenced the findings.

In conclusion, we show an association between anatomical extent of PE, RVD, and the risk of recurrence. Because the indirect meta-analysis suggests a possible benefit of a heparin lead-in, future DOAC studies evaluating the role of heparin might focus on PE patients with more extensive disease.

\section{Authors' Contributions}

M.P.A.B., H.R.B., M.F.M., and J.I.W. were responsible for conception and design or analysis and interpretation of 
data, or both. All authors had full access to the data and contributed to drafting of the manuscript or revising it critically for important intellectual content and provided final approval of the manuscript submitted.

\section{Funding}

This study was performed without financial support. Four authors are employees of the sponsor of the original Hokusai-VTE study, Daiichi Sankyo, who provided data from the Hokusai-VTE study without restrictions.

\section{Conflicts of Interest}

M.P.A.B. has nothing to disclose. H.R.B. reports grants and personal fees from Bayer, Boehringer Ingelheim, BristolMyers Squibb, Daiichi Sankyo, GlaxoSmithKline, Ionis Pharmaceuticals, Pfizer, Roche, Sanofi, and ThromboGenics outside the submitted work. M.F.M. is an employee of Daiichi-Sankyo Pharma Development. W.A. reports grants, personal fees, and nonfinancial support from Bayer, Boehringer Ingelheim, Daiichi Sankyo, and Stago; and personal fees from Bristol-Myers Squibb, Pfizer, and Aspen, outside the submitted work. C.Z.C. is an employee of Daiichi-Sankyo, Inc. A.T.C. reports grants from DaiichiSankyo Pharma Development during the conduct of the study; grants and personal fees from Bayer, Bristol-Myers Squibb, and Pfizer; and personal fees from Boehringer Ingelheim, Janssen, Johnson \& Johnson, Portola, Sanofi, and X01 outside the submitted work. N.v.E. reports a personal fee from Pfizer outside the submitted work. M.A. G. is an employee of Daiichi-Sankyo Pharma Development. A.P.M. has nothing to disclose. G.R. reports personal fees from Daiichi-Sankyo and Itreas during the conduct of the study; and personal fees from Bayer, Boehringer Ingelheim, Bristol-Myers Squibb, Eli Lilly, Ionis Pharmaceuticals, Janssen, Pfizer, and Portola outside the submitted work. A.S. reports grants from Daiichi-Sankyo Pharma Development during the conduct of the study; and grants from Ionis Pharmaceuticals outside the submitted work. T.V. reports grants from Daiichi-Sankyo Pharma Development during the conduct of the study. P.V. reports grants and personal fees from Daiichi-Sankyo Pharma Development during the conduct of the study; and grants from Leo Pharma; grants and personal fees from Boehringer Ingelheim, Sanofi, and ThromboGenics; and personal fees from Bayer outside the submitted work. P.S.W. reports personal fees from Bayer and Daiichi Sankyo; and grants from Bristol-Myers Squibb and Pfizer outside the submitted work. G.Z. is an employee of Daiichi-Sankyo Pharma Development. J.I.W. reports personal fees from Bayer, Boehringer Ingelheim, BristolMyers Squibb, Daiichi Sankyo, Ionis Pharmaceuticals, Janssen, Pfizer, Novartis, and Portola outside the submitted work.

\section{References}

1 Yeh CH, Gross PL, Weitz JI. Evolving use of new oral anticoagulants for treatment of venous thromboembolism. Blood 2014;124(07): $1020-1028$
2 Kearon C, Akl EA, Ornelas J, et al. Antithrombotic therapy for VTE disease: CHEST guideline and expert panel report. Chest 2016; 149(02):315-352

3 Schulman S, Kearon C, Kakkar AK, et al; RE-COVER Study Group. Dabigatran versus warfarin in the treatment of acute venous thromboembolism. N Engl J Med 2009;361(24):2342-2352

4 Schulman S, Kakkar AK, Goldhaber SZ, et al; RE-COVER II Trial Investigators. Treatment of acute venous thromboembolism with dabigatran or warfarin and pooled analysis. Circulation 2014;129 (07):764-772

5 Bauersachs R, Berkowitz SD, Brenner B, et al; EINSTEIN Investigators. Oral rivaroxaban for symptomatic venous thromboembolism. N Engl J Med 2010;363(26):2499-2510

6 Büller HR, Prins MH, Lensin AW, et al; EINSTEIN-PE Investigators. Oral rivaroxaban for the treatment of symptomatic pulmonary embolism. N Engl J Med 2012;366(14):1287-1297

7 Agnelli G, Buller HR, Cohen A, et al; AMPLIFY Investigators. Oral apixaban for the treatment of acute venous thromboembolism. N Engl J Med 2013;369(09):799-808

8 Büller HR, Décousus H, Grosso MA, et al; Hokusai-VTE Investigators. Edoxaban versus warfarin for the treatment of symptomatic venous thromboembolism. N Engl J Med 2013;369(15): 1406-1415

9 Rudd KM, Phillips EL. New oral anticoagulants in the treatment of pulmonary embolism: efficacy, bleeding risk, and monitoring. Thrombosis 2013;2013:973710

10 Gouin B, Blondon M, Jiménez D, et al; RIETE investigators. Clinical prognosis of nonmassive central and noncentral pulmonary embolism: a registry-based cohort study. Chest 2017;151(04): 829-837

11 Trujillo-Santos J, Di Micco P, Dentali F, et al; RIETE Investigators. Real-life treatment of venous thromboembolism with direct oral anticoagulants: the influence of recommended dosing and regimens. Thromb Haemost 2017;117(02):382-389

12 Raskob G, Büller H, Prins M, et al; Hokusai-VTE Investigators. Edoxaban for the long-term treatment of venous thromboembolism: rationale and design of the Hokusai-venous thromboembolism study-methodological implications for clinical trials. J Thromb Haemost 2013;11(07):1287-1294

13 van Es N, Coppens M, Schulman S, Middeldorp S, Büller HR. Direct oral anticoagulants compared with vitamin $\mathrm{K}$ antagonists for acute venous thromboembolism: evidence from phase 3 trials. Blood 2014;124(12):1968-1975

14 Goldhaber SZ, Schellong S, Kakkar A, et al. Treatment of acute pulmonary embolism with dabigatran versus warfarin. A pooled analysis of data from RE-COVER and RE-COVER II. Thromb Haemost 2016;116(04):714-721

15 Klok FA, Mos IC, Huisman MV. Brain-type natriuretic peptide levels in the prediction of adverse outcome in patients with pulmonary embolism: a systematic review and meta-analysis. Am J Respir Crit Care Med 2008;178(04):425-430

16 Konstantinides SV, Torbicki A, Agnelli G, et al; Task Force for the Diagnosis and Management of Acute Pulmonary Embolism of the European Society of Cardiology (ESC). 2014 ESC guidelines on the diagnosis and management of acute pulmonary embolism. Eur Heart J 2014;35(43):3033-3069, 3069a-3069k

17 Lankeit $M$, Jiménez $D$, Kostrubiec $M$, et al. Validation of $\mathrm{N}$ terminal pro-brain natriuretic peptide cut-off values for risk stratification of pulmonary embolism. Eur Respir J 2014;43 (06):1669-1677

18 Jiménez D, Kopecna D, Tapson V, et al; On Behalf Of The Protect Investigators. Derivation and validation of multimarker prognostication for normotensive patients with acute symptomatic pulmonary embolism. Am J Respir Crit Care Med 2014;189(06): 718-726

19 Sanchez O, Trinquart L, Caille V, et al. Prognostic factors for pulmonary embolism: the prep study, a prospective multicenter cohort study. Am J Respir Crit Care Med 2010;181(02):168-173 
20 Brekelmans MP, Ageno W, Beenen LF, et al. Recurrent venous thromboembolism in patients with pulmonary embolism and right ventricular dysfunction: a post-hoc analysis of the HokusaiVTE study. Lancet Haematol 2016;3(09):e437-e445

21 Ribeiro A, Juhlin-Dannfelt A, Brodin LA, Holmgren A, Jorfeldt L. Pulmonary embolism: relation between the degree of right ventricle overload and the extent of perfusion defects. Am Heart J 1998;135(5, Pt 1):868-874

22 Wong LF, Akram AR, McGurk S, Van Beek EJ, Reid JH, Murchison JT. Thrombus load and acute right ventricular failure in pulmonary embolism: correlation and demonstration of a "tipping point" on CT pulmonary angiography. Br J Radiol 2012;85(1019):1471-1476 